\title{
LA GUÍA DOCENTE EN LA PLANIFICACIÓN Y DESARROLLO DE LA ENSEÑANZA UNIVERSITARIA ${ }^{1}$
}

\author{
Pedro Ricardo Álvarez-Pérez \\ David López-Aguilar \\ Rocío Peña-Vázquez \\ María Olga González-Morales \\ Universidad de La Laguna- ULL (España)
}

\begin{abstract}
Resumen
El proceso intencional que se viene desarrollando en los últimos tiempos en la enseñanza universitaria cuenta con un referente fundamental, la guía docente, que debe estar en sintonía con los principios que orientan el proceso de convergencia europea y recoger la planificación de la enseñanza que realiza el profesorado para gestionar el aprendizaje del alumnado. Por eso, la planificación docente constituye un elemento de calidad, dado que para lograr un buen aprendizaje en el alumnado se requiere una buena guía del trabajo que tiene que desarrollar en cada materia. Sobre la base de este planteamiento, se llevó a cabo un estudio de corte cualitativo, basado en entrevistas al profesorado y focus group con informantes claves vinculados a la enseñanza universitaria, con el objetivo de valorar la utilidad de la guía docente como recurso didáctico. Los resultados pusieron de manifiesto que la guía docente es un instrumento básico en el modelo actual de la enseñanza universitaria. Sin embargo, tiene una función más administrativa que didáctica, ya que es un recurso que se utiliza para los procesos de acreditación de titulaciones y para informar al alumnado al inicio del curso de los aspectos claves de las asignaturas, pero no se integra en la dinámica diaria del proceso formativo como una guía del aprendizaje.
\end{abstract}

Palabras clave: Guía docente; planificación de la enseñanza; gestión del aprendizaje; modelo formativo.

\section{THE TEACHING GUIDE IN THE PLANNING AND DEVELOPMENT OF UNIVERSITY EDUCATION}

\begin{abstract}
.
The intentional process that has been developing in recent times in university education has a fundamental reference, the teaching guide, which must be in line with the principles that guide the European convergence process and collect the planning of the teaching carried out by the teachers to manage student learning. Therefore, teacher planning is an element of quality, given that to achieve good learning in students requires a good guide of the work you have to develop in each subject. On the basis of this approach, a qualitative study was carried out, based on interviews with teachers and focus groups with key informants linked to university education, with the aim of assessing the usefulness of the teaching guide as a teaching resource. The results showed that the teaching guide is a basic instrument in the current model of university education. However, it has a more administrative than didactic function, since it is a resource that is used for the accreditation processes of degrees and to inform the students at the beginning of the course of the key aspects of the subjects, but it is not integrated into the dynamics daily of the formative process as a guide to learning.
\end{abstract}

Keywords: Teaching guide; teaching planning; learning management; formative model

\footnotetext{
${ }^{1}$ Este artículo deriva de un proyecto financiado por la Universidad de La Laguna (Convocatoria de Proyectos de Innovación Educativa, Vicerrectorado de Docencia, curso 2016/2017).
} 


\title{
GUIA DE ENSINO NO PLANEJAMENTO E DESENVOLVIMENTO DA EDUCAÇÃO UNIVERSITÁRIA
}

\begin{abstract}
Resumo
O processo intencional que tem vindo a desenvolver nos últimos tempos no ensino universitário tem uma referência fundamental, o guia de ensino, que deve estar em consonância com os princípios que orientam o processo de convergência europeu e o planejamento do ensino realizado pelo professores para gerenciar a aprendizagem dos alunos. Por esta razão, o planejamento de professores é um elemento de qualidade, dado que, para alcançar uma boa aprendizagem nos alunos, é necessário um bom guia do trabalho que deve ser desenvolvido em cada assunto. Com base nessa abordagem, foi realizado um estudo qualitativo, com base em entrevistas com professores e grupos focais com informantes chave ligados ao ensino universitário, com o objetivo de avaliar a utilidade do guia de ensino como recurso de ensino. Os resultados mostraram que o guia de ensino é um instrumento básico no modelo atual de educação universitária. No entanto, tem uma função mais administrativa do que didática, uma vez que é um recurso que é usado para os processos de acreditação de graus e para informar os alunos no início do curso dos principais aspectos dos assuntos, mas não está integrado na dinâmica diariamente do processo formativo como um guia para aprender.
\end{abstract}

Palavras-chave: Guia de ensino; planejamento de ensino; gestão de aprendizagem; modelo formativo.

\section{Introducción}

Con la implantación del Espacio Europeo de Educación Superior (EEES) se ha venido convergiendo hacia un modelo formativo común entre los sistemas educativos universitarios de los distintos países europeos. Entre las novedades básicas que se incorporan con este enfoque, se establece un mecanismo de créditos y un sistema comparable de titulaciones para promover la movilidad de los estudiantes. Es en este marco general de la Convergencia Europea (Declaración de Bolonia, 1999) el profesorado universitario tiene un papel clave a la hora de facilitar el proceso de aprendizaje del alumnado, fomentando en los estudiantes el "aprender a aprender" (Arias-Gundín, Fidalgo y García, 2008; Sanz, Ruiz y Pérez, 2014). Para lograr este objetivo prioritario de la enseñanza universitaria, se debe reforzar la planificación, tanto de las titulaciones que conforman la propia oferta formativa, como de las asignaturas y materias que la integran, de modo que las guías docentes sean un referente básico para el estudiante (Fidalgo y García, 2007; Cano, Tomás, García, Bañón, Riquelme y Murcia, 2016). Morón y Pedrero (2012, p. 375) plantean que, a través de la guía docente, se consigue optimizar "los procesos de planificación docente de forma que se facilite la visibilidad y comparabilidad de los programas".

Por estos motivos, la guía docente se ha convertido en los últimos tiempos en un recurso estratégico de la organización y planificación de los títulos universitarios. La guía docente sitúa las asignaturas en el marco general de la titulación y recoge la contribución que desde cada materia se hace al desarrollo del perfil profesional del título. Fernández y Fernández (2007, p. 55) plantean que la guía docente debe especificar "cuáles son los objetivos que se pretenden con la inclusión de la materia en un plan de estudios que conduce a un perfil profesional".

La importancia que ha alcanzado la guía docente en el modelo formativo universitario actual, exige que se tome muy en cuenta los procesos de planificación de la enseñanza y se reconozca esta tarea entre las funciones a desempeñar por los docentes universitarios (Díez, Pacheco y García, 2008; García, 2014; Marcelo, Yot, Mayor, 
Sánchez, Murillo, Rodríguez y Pardo, 2014). En concreto, Mas (2012) reconoce que entre las competencias referidas a la función docentes se encuentra el diseño de las guías docentes, que se han de diseñar de acuerdo a las necesidades del contexto y al perfil profesional de la titulación. Requiere, por parte del profesorado, reflexión, previsión y preparación del proceso de enseñanza-aprendizaje.

Sánchez, Ruiz y Pascual (2011, p. 53), al referirse a la guía docente, señalan que es "una delineación básica de los principios que regirán el desarrollo de la materia o módulo. La guía significa estructura en el mismo paquete [...] una selección y ordenación de contenidos académicos y [...] una oferta didáctica; esto es, cómo van a ser trabajados esos contenidos y cómo van a ser evaluados los estudiantes". La guía docente es un instrumento que sirve para planificar, de manera detallada y estructurada, cualquier asignatura o módulo, ajustando la programación a los principios y directrices del EEES. Se trata, por tanto, de un recurso del que no se puede prescindir y que, como resaltan García y De la Cruz (2014), ayuda a optimizar las tareas del profesorado y el alumnado universitario.

Para Zabalza (2003), la guía docente es un recurso de planificación a nivel micro (de asignatura, no de titulación) cuyo objetivo prioritario es informar y orientar a los estudiantes acerca del proceso de estudio. García (2014, p.3) entiende la guía docente "como el documento que orienta el estudio, acercando a los procesos cognitivos del alumno el material didáctico, con el fin de que pueda trabarlo de manera autónoma". Esta planificación requiere de un cuidadoso proceso de selección de contenidos para que el estudiante alcance los aprendizajes esperados (Salinas y Cotillas, 2005). Por tanto, en la guía docente se detallan los elementos relevantes de una asignatura. En palabras de García y Deltell (2012, p. 358) es el "eje vertebral del modelo formativo a desarrollar". Aparecen descritos los objetivos formativos, las competencias que se adquieren, el programa, la metodología, la bibliografía y el catálogo de técnicas docentes, de actividades académicas y de métodos de evaluación.

El concepto de guía docente, como muchos otros referentes del modelo formativo universitario, ha sufrido cambios con la adaptación al modelo del EEES. Para el profesorado, al elaborar la guía docente debe relacionar los objetivos del aprendizaje con las competencias que se pretenden desarrollar en una materia, a través de determinadas actividades formativas y empleando la evaluación como elemento de seguimiento y control del aprendizaje. Esta planificación se ocupa de aspectos como la adecuada distribución de la carga de trabajo del alumnado, la organización temporal de las actividades formativas, incluyendo entre éstas las pruebas de evaluación, los materiales docentes y los contenidos, los plazos de entrega de trabajos e informes, la metodología de evaluación y todo lo que el estudiante necesita conocer para superar una asignatura.

Desde esta perspectiva, la guía docente es una herramienta fundamental para la orientación y la tutoría académica que realiza el profesorado en las materias que imparte. Sin embargo, la puesta en práctica de un enfoque más personalizado de la enseñanza tropieza, como destacan González, Gilar, Mañas y Bermejo (2005), con problemas que presenta la educación superior actualmente, como es la elevada ratio de alumnado por grupo y asignatura. Precisamente, está fue una de las limitaciones importantes que encontraron los autores en la experiencia que desarrollaron sobre los elementos que configuran la guía docente, como material de trabajo en la docencia universitaria. Insisten en que para el desarrollo de una docencia que se adapte al ritmo de aprendizaje de los estudiantes, que se pueda llevar a cabo una evaluación en 
profundidad de las asignaturas, para que se pueda guiar el proceso de aprendizaje, se debe contar con condiciones más favorables en cuanto al tamaño de los grupos, mayor número de horas de dedicación del profesorado a cada estudiante, mejores recursos para un contacto permanente y personalizado, etc.

Para los estudiantes, la guía docente es un documento de referencia, que les permite optimizar su trabajo y obtener el máximo aprovechamiento del proceso de enseñanza-aprendizaje. Salinas y Cotillas (2005) resumen las funciones principales de las guías docentes:

- Especificar la oferta docente referida a una asignatura o módulo. Concretamente, es la forma en la que la universidad hace pública su oferta formativa, tanto en lo que se refiere a los contenidos disciplinares de la asignatura, como a los resultados esperados del aprendizaje, así como las actividades de enseñanza-aprendizaje y los criterios de evaluación.

- Informar al alumnado de lo que se pretende que aprenda, cómo se va a desarrollar el proceso de enseñanza-aprendizaje, bajo qué condiciones y cómo será evaluado.

- Recoger el compromiso del personal docente sobre diferentes cuestiones, como son los contenidos, los métodos docentes y de evaluación, etc. que se irán desarrollando a lo largo de un curso. Además, es un documento público en el que se hace referencia a la estructura de una oferta académica particular.

- Garantizar que la materia cumple con los requisitos y funciones que le son asignadas en el plan de estudios.

En el estudio de García y Deltell (2012), se concluyó, en primer lugar, que se trata de un recurso formativo que ofrece pautas y orientaciones para que el alumnado sea capaz de enfrentar adecuadamente su proceso de aprendizaje; por otra parte, que el estudiante debe asumir su papel protagonista y, en este rol, la guía docente ocupa un importante lugar para orientar su trayectoria académica; y, finalmente, que docente y alumno deben asumir que la formación es un proceso compartido entre ambos. Compartimos la idea de Sánchez, Ruiz, y Pascual (2001, p. 61) cuando señalan que la planificación de la enseñanza es indispensable para que los estudiantes aprendan a aprender, pero para que esto sea posible "tanto el alumno como el profesor tienen que darse cuenta que deben efectuar una renovación de la metodología que habitualmente utilizan y planificar el proceso de enseñanza aprendizaje teniendo en cuenta como punto de referencia el trabajo que el alumno debe realizar tanto de forma presencial como no presencial"

Aunque la guía docente se presenta como un elemento fundamental en el modelo formativo actual de la enseñanza universitaria, se viene cuestionando si se trata de un recurso de utilidad para guiar el proceso de enseñanza y aprendizaje de las materias o, si por el contrario, es una herramienta que cumple únicamente con una función administrativa que emplean las instituciones de educación superior, para cumplir con los procesos de verificación y acreditación exigidos por la Agencia Nacional de Evaluación de la Calidad y Acreditación (ANECA). Especial relevancia tiene el programa VERIFICA, con el que se evalúa las propuestas de planes de estudio de títulos diseñados en consonancia con el EEES. Para ello, las universidades deben presentar una memoria de verificación de cada título, que debe ajustarse a los criterios y estándares de calidad de la ANECA antes de su implantación. En la memoria de verificación (o, en su caso, su posterior modificación) se recoge la descripción y estructura de los títulos 
universitarios oficiales, desde la justificación del título, las competencias, los sistemas de calidad, la planificación de la enseñanza, etc.

Para analizar el modelo de guía docente que se viene empleando en la Universidad de La Laguna (ULL) tras la incorporación del modelo del EEES y valorar su importancia como recurso para guiar el proceso formativo del alumnado en el marco de la convergencia europea, se realizó un estudio a partir de las opiniones de decanos, coordinadores de calidad, directores de Departamento, profesorado, alumnado, técnicos de evaluación de la calidad y asesores jurídicos. De manera más específica, los objetivos del estudio fueron:

- Valorar la utilidad que tiene la guía docente como recurso de la enseñanza universitaria.

- Analizar el proceso de diseño de la guía docente.

- Valorar el contenido y estructura de la guía docente.

- Valorar el grado de cumplimiento de la propuesta formativa recogida en la guía docente.

\section{Método}

\section{Participantes}

En este estudio, de corte cualitativo, se empleó una entrevista semiestructurada dirigida a docentes de la Universidad de La Laguna y un grupo de discusión con diferentes informantes clave.

Con la finalidad de seleccionar a los participantes que colaboraron en la entrevista, se empleó un muestro intencional de expertos. El requisito establecido para participar era ser docente universitario en cualquiera de las ramas de conocimiento impartidas en la Universidad de La Laguna. Las valoraciones aportadas por los docentes universitarios en esta técnica de recogida de información se consideraron relevantes por su experiencia en torno a los objetivos que se pretendían cubrir con este trabajo.

Como se puede observar en la tabla 1, participaron 10 docentes, de los cuales 4 eran hombres $(40 \%)$ y 6 mujeres $(60,0 \%)$, distribuidos de manera equitativa en las distintas ramas de conocimiento: artes y humanidades $(20,0 \%)$, ciencias $(20,0 \%)$, ciencias de la salud $(20,0 \%)$, ciencias sociales y jurídicas $(20,0 \%)$ e ingeniería y arquitectura $(20,0 \%)$.

Tabla 1

Características de la muestra participante en la entrevista semiestructurada

\begin{tabular}{lll}
\hline \multicolumn{3}{c}{ Número de docentes participante en la entrevista semiestructurada $=10(\mathrm{n}=10)$} \\
\hline Rama de conocimiento & Codificación & Género \\
\hline Artes y humanidades & EAH1 & Mujer \\
\cline { 2 - 3 } & EAH2 & Hombre \\
\hline
\end{tabular}




\begin{tabular}{lll} 
& EC1 & Mujer \\
\cline { 2 - 3 } & EC2 & Mujer \\
\hline Ciencias de la salud & ECS1 & Hombre \\
\cline { 2 - 3 } & ECS2 & Mujer \\
\hline Ciencias sociales y jurídicas & ECSJ1 & Mujer \\
\cline { 2 - 3 } & ECSJ2 & Hombre \\
\hline Ingeniería y arquitectura & EIA1 & Hombre \\
\cline { 2 - 3 } & EIA2 & Mujer \\
\hline
\end{tabular}

En cuanto al grupo de discusión, se contó con la participación de 8 informantes clave, distribuidos de la siguiente manera: un estudiante universitario (EU), un técnico de la Unidad de evaluación institucional de la calidad perteneciente al Vicerrectorado de Docencia (UCAL), un decano (DEC), dos profesores universitarios (PU1; PU2), un miembro de la asesoría jurídica del Vicerrectorado de Estudiantes (AJUR), un director de Departamento (DIDE) y un moderador no participante (tabla 2). Los miembros que conformaron el grupo de discusión tenían una relación directa con el diseño, implementación y evaluación de las guías docentes en el contexto de la enseñanza universitaria, por lo que se consideró necesaria su participación para completar la visión aportada por los docentes en las entrevistas.

Tabla 2.

Características de los informantes clave participante en el grupo de discusión

Número de docentes participante en la entrevista semiestructurada $=10(n=10)$

\begin{tabular}{ll|l}
\hline Participante & Codificación & Género \\
\hline Estudiante Universitario & EU & Mujer \\
\hline Técnico de la Unidad de Calidad & UCAL & Mujer \\
\hline Decano & DEC & Hombre \\
\hline Profesor universitario 1 & PU1 & Hombre \\
\hline Profesor universitario 2 & PU2 & Mujer \\
\hline Responsable de Asesoría Jurídica & AJUR & Mujer \\
\hline Director de departamento & DIDE & Hombre \\
\hline Moderador no participante & & Mujer \\
\hline
\end{tabular}

\section{Instrumentos de recogida de información}

Para la recogida de datos se empleó la entrevista semiestructurada y el grupo de discusión anteriormente descritos. La estrategia cualitativa, como plantean Huber 
(1992) y Ercikan y Roth (2006), ofrece un conocimiento preciso sobre la realidad objeto de análisis desde una perspectiva crítica y reflexiva. Además, el empleo de diferentes estrategias de recopilación de datos, permite triangular la información obtenida, aumentando de esta manera, los niveles de credibilidad y validez de los resultados.

La entrevista es una herramienta que permite recoger los relatos para interpretar una determinada realidad. Para diseñar el guión de la entrevista que orientó el desarrollo de las sesiones, y con la finalidad de aumentar su validez y fiabilidad, se tuvieron en cuenta:

- Las propuestas planteadas por Grinnel (1997) con relación al tipo de preguntas que se deben emplear en las entrevistas semiestructuradas: abiertas, de ejemplificación, estructurales, de contraste, etc.

- Los planteamientos de Rogers y Bouey (2005), quienes sostienen que las preguntas establecidas deben estar adaptadas a las características de los participantes, se debe generar un adecuado clima para el desarrollo de la entrevista (establecimiento del rapport), se debe ajustar el lenguaje y las normas a las condiciones de los entrevistados, etc.

Para planificar el grupo de discusión, se tuvieron en cuenta las directrices propuestas por Suárez (2005), con relación al número de participantes que debe colaborar como informantes clave, los criterios de selección de las personas participantes, el tiempo de administración recomendado de esta prueba, etc.

El guión definitivo de la entrevista (1) quedó configurado con un total de 11 preguntas. Por otra parte, el guión para el debate grupal (2) estaba compuesto por 21 preguntas. El guión de preguntas, tanto de la entrevista como del grupo de discusión, giró en torno a las siguientes dimensiones: utilidad de la guía docente (a), diseño de la guía docente (b), contenido y estructura de la guía docente (c) y cumplimiento de la propuesta recogida en la guía docente (d). Estas dimensiones objeto de análisis fueron confirmadas y validadas mediante el uso del Atlas.Ti (tabla 3).

Tabla 3

Dimensiones y variables objeto de análisis

\begin{tabular}{clll}
\hline Dimensión & Definición & Código \\
\cline { 2 - 3 } & $\begin{array}{l}\text { Recogía información relacionada con el uso y la } \\
\text { utilidad que se le concedía a la guía docente en el } \\
\text { marco de la enseñanza universitaria. }\end{array}$ & UTIGD \\
\hline Diseño de la guía docente & $\begin{array}{l}\text { Recogía información sobre cómo era el proceso de } \\
\text { diseño y construcción del contenido de la guía } \\
\text { docente. }\end{array}$ & DIGD \\
\hline $\begin{array}{c}\text { Contenido y estructura de } \\
\text { la guía docente }\end{array}$ & $\begin{array}{l}\text { Valoraba aspectos relacionados con la estructura y el } \\
\text { contenido que se incorporaba en la guía docente. }\end{array}$ & ESGD \\
\hline $\begin{array}{c}\text { Cumplimiento de la } \\
\text { propuesta recogida en la } \\
\text { guía docente }\end{array}$ & $\begin{array}{l}\text { Pretendía analizar en qué medida los aspectos } \\
\text { introducidos en la guía docente se desarrollaban a lo } \\
\text { largo del desarrollo de las materias. }\end{array}$ & CUMGD \\
\hline
\end{tabular}




\section{Procedimiento de recogida de datos y análisis de información}

Tras el proceso de construcción y diseño de las herramientas, se llevó a cabo la recogida de información. El procedimiento que se empleó para la entrevista fue el siguiente:

- Cada entrevistador ( $\mathrm{n}=5$; un entrevistador por área de conocimiento) se puso en contacto con los profesores de las áreas de conocimiento objeto de análisis con la intención de explicarles las finalidades del estudio y solicitar su participación voluntaria en una entrevista semiestructurada.

- Una vez las personas aceptaron participar como informantes clave en la entrevista, fueron convocadas a una hora, fecha y lugar que, previamente fue acordada entre entrevistador y entrevistado.

Para la elaboración de la sesión del grupo de discusión, se fijó una fecha, hora y lugar a conveniencia entre los informantes clave y el moderador. Además, los días previos al grupo de discusión, se envió un correo electrónico a cada uno de los participantes recordándoles los detalles de la cita e informándoles sobre las cuestiones básicas que se iban a tratar.

Siguiendo las propuestas de McMillan y Schumacher (2005) y Hernández, Fernández-Collado y Baptista (2006) para aumentar la validez y fiabilidad del proceso y de los resultados, en la aplicación de las entrevistas y la celebración del grupo de discusión, se emplearon diferentes estrategias como: participación de distintos investigadores en las sesiones de recogida de datos, anotación de las incidencias que eran de interés para los objetivos propuestos en el estudio, grabación digital de las pruebas, realización de transcripciones narrativas verbatim de la información obtenida y proceso de codificación y análisis realizado por varios investigadores.

\section{Análisis e interpretación de la información}

Para el tratamiento de la información se han seguido las propuestas de Miles y Huberman (1994) y Strauss y Corbin (2002), en cuanto a la reducción de los datos, la disposición y transformación, y la verificación de los resultados. Como apoyo metodológico al análisis de los datos cualitativo, se utilizó el software informático Atlas.Ti 6. En concreto, se siguió el siguiente procedimiento:

- Construcción de una unidad hermenéutica, donde se incluyeron los documentos primarios de las entrevistas realizadas y del grupo de discusión celebrado.

- Reducción de la información, identificando las citas más relevantes que ofrecían respuesta a los objetivos propuestos en el estudio.

- Codificación de los relatos obtenidos a partir de las categorías previamente establecidas y de las dimensiones que emergieron del análisis de los documentos primarios.

\section{Resultados}

Los resultados del estudio se presentan agrupados en torno a las dimensiones planteadas en el trabajo.

\section{Utilidad de la guía docente}


Uno de los objetivos fundamentales del trabajo realizado era determinar qué utilidad y qué usos se hacía de la guía docente en el contexto de la Universidad de La Laguna. A partir de los relatos ofrecidos por el profesorado en las entrevistas y por los informantes clave en el grupo de discusión se pudieron identificar tres utilidades básicas de la guía docente.

En primer lugar, una parte del profesorado entrevistado (30,0\%) reflejó que la guía docente era un recurso fundamentalmente administrativo y se empleaba poco a lo largo del proceso de enseñanza aprendizaje (tabla 4). En este sentido, uno de los relatos recogidos indicó que "no es una herramienta que yo emplee de manera cotidiana en el aula" (EIA1), ya que "la guía, tal y como está recogida es un documento que está pensando para la burocracia, para que después, cuando vengan las acreditaciones, encaje en la memoria de verificación (...) Es un instrumento de control para las evaluaciones externas que hagan del título, no sirve para otra cosa” (ECSJ2).

Tabla 4

Utilidad de la guía docente

\begin{tabular}{lc}
\hline & Porcentaje \\
\hline Documento administrativo & $30,0 \%$ \\
\hline Documento formativo & $50,0 \%$ \\
\hline Documento administrativo y formativo & $20,0 \%$ \\
\hline
\end{tabular}

Estas opiniones fueron compartidas por los participantes en el grupo de discusión. Concretamente, el responsable de la unidad técnica de calidad afirmó que "la herramienta estrella del proceso de seguimiento y acreditación de titulaciones es la guía docente, puesto que es el mapa de carretera que utilizan los evaluadores" (UCAL). Opinión que fue reforzada por el decano, quien señaló que era básicamente un "registro administrativo que después va a tener una repercusión y un recorrido en el proceso de acreditación” (DEC).

Por otra parte, el 50,0\% de los docentes entrevistados, coincidió al defender que la guía docente debe servir para orientar la enseñanza, permitiendo planificar diferentes aspectos que están en estrecha conexión con el proceso de enseñanza-aprendizaje del alumnado. Los entrevistados entendieron que "la guía docente es el mapa de la asignatura, es la hoja de ruta de la asignatura" (EIA2). Coincidiendo con estas argumentaciones, el decano que participó en el grupo de discusión afirmó que "las guías docentes han contribuido de forma determinante a instaurar una cultura de calidad de la docencia que no existía antes, cuestiones como, por ejemplo: qué debo enseñar, una programación temporal, una definición lo más precisa y rigurosa de la evaluación" (DEC). Con relación a estas valoraciones, se pronunció uno de los docentes participantes en la entrevista señalando que "cierto es que, para algunos profesores, que no tenían ningún tipo de programación, (...) les ha venido bien, para pensar un poquito en la asignatura" (ECSJ2)

Aunque no se ha extendido de manera generalizada el uso de la guía docente como recurso de la enseñanza, muchos docentes hicieron un uso diario del programa con sus estudiantes. A modo de ejemplo, uno de los profesores entrevistados relató 
cómo empleaba este recurso de manera integrada en su asignatura: "todas las clases empiezan diariamente abriendo la guía docente, indicándoles en qué parte de la guía docente estamos; esta semana estamos en el tema cuatro y estamos viendo el apartado de meta-programación" (EIA2). Sin embargo, la mayor parte de los docentes entrevistados, limitó el uso de este recurso "al primer día de clase; es decir, la presento, yo resumo lo que viene en todos los apartados, los contenidos que vamos a ver, la metodología y, evidentemente, el sistema de evaluación” (EAH1).

Desde la perspectiva del representante de la asesoría jurídica que participó, el uso que hicieron los estudiantes de la guía docente se reducía básicamente a "hacerse una idea a principio de curso del temario, de en qué consiste esa asignatura, aquel que tenga un interés digamos a priori de antes de matricularse de qué va esta asignatura, que con el nombre nada más a lo mejor no puedo definir los contenidos, y desde luego en el aspecto de evaluación" (AJUR).

Confirmando este argumento, el estudiante que participó en el grupo de debate insistió en la idea de que la guía docente es un recurso de escasa utilidad para el proceso formativo del alumnado. En este sentido destacó que "no le prestamos mucha atención a las guías docentes, porque después, por ejemplo, establecen en la evaluación un examen oral o un examen escrito, pero a lo mejor nunca llegamos a ese examen" (EU). Según este estudiante, la utilidad y uso que hacía de la guía docente se centraba básicamente en conocer "quién es el profesor, las tutorías o el horario" (EU).

Por último, hubo un grupo de personas que participó en la sesión de debate que consideró la guía docente como un recurso que, por una parte, era de carácter administrativo y, por otra, servía para guiar y orientar el proceso de enseñanza. En palabras de uno de los participantes en el grupo de discusión, la guía docente era un "un proceso administrativo ineludible, pero también para el alumno y uno mismo es una herramienta para el buen desarrollo del curso, sobre todo lo que es el ajuste de los contenidos al cronograma; a mi me parece interesante" (EAH2).

\section{Diseño de la guía docente}

Otro de los aspectos que se valoró en este estudio fue el proceso que se siguió para la elaboración y el diseño de la guía docente. De los testimonios que ofrecieron los participantes en el estudio, destacó la rigidez que encontraron los docentes a la hora de construir su programa formativo. Es decir, existía poco espacio para reflejar la propuesta y el planteamiento pedagógico de las asignaturas. Ante esta situación, manifestaron que "he notado que cuando la estoy redactando hay cosas que me resultan algo rígidas, que a lo mejor debería dar más posibilidad de editar" (ECS2). Este planteamiento, no permitió recoger, por ejemplo, "el esfuerzo que yo le dedico a la asignatura; es decir, yo destino mucho tiempo a preparar la docencia cada año, a actualizarla. En cuanto he terminado con una asignatura, ya estoy mirando de qué modo la actualizo y de qué modo incorporo este o aquel otro modelo o aquel otro detalle, aquella investigación” (ECS1).

Las valoraciones de los participantes resaltaron la importancia que tenían las memorias de verificación de las titulaciones a la hora de determinar el desarrollo de las asignaturas. La memoria de verificación del título concreta aspectos que los docentes tienen que asumir y desarrollar, sin poder cambiar los apartados esenciales de la guía docente hasta que no se modifique. Esta realidad, ofrece una escasa capacidad de maniobra para que el docente pueda incorporar en las guías elementos actualizados e innovadores vinculados a su proceso formativo. Así, uno de los profesores que participó 
en el grupo de discusión indicó que "el sistema de evaluación es lo que más puedes, a lo mejor, variar un poco. Pero tampoco tanto, porque está muy regulado por la memoria de verificación" (EAH1). Otro de los docentes señaló que "quizás donde mayor modificación hay con respecto a otros años es en la evaluación. Luego lo que son las competencias no, porque las competencias vienen establecidas por una orden ministerial, que es una titulación con repercusión profesional, ahí no tenemos margen y en el desarrollo de los contenidos, son los que tenemos a lo largo de los años, no modificamos mucho" (EIA1). En definitiva, más que sustituir o incluir cualquier aspecto en la guía docente, "sería la memoria de verificación la que tendrías que modificar, porque lo que yo no puedo modificar es una competencia en la guía docente si no está modificada en la memoria de verificación” (EC1).

La existencia de un modelo de guía docente con apartados e información prescrita, quedó justificada por el representante de la unidad de calidad que participó en el grupo de discusión al indicar que "todo lo que tiene que ver con competencias, todas esas cuestiones... se las damos ya cerrados porque (...) detectamos que no se correspondía nada con lo establecido" (UCAL).

Desde la perspectiva del profesorado entrevistado, la memoria de verificación es "también un documento de planificación educativa, que se tendría que estar actualizando constantemente" (ECSJ2). Para que esto funcione de manera efectiva y se consigan los ajustes oportunos y necesarios, el proceso de actualización de las memorias de verificación tendría que ser más ágil y rápido. Solo de esta manera se podrían corregir los desajustes entre lo que figura en la memoria de verificación y lo que ocurre realmente en el desarrollo de una asignatura. En este sentido, algunos participantes en el grupo de discusión, reclamaron mayor capacidad para adecuar el programa de la asignatura a las características del curso y del alumnado, dado que es muy difícil prever con antelación lo que va a ocurrir durante el desarrollo de la asignatura. Algunos de los relatos recogidos apuntaron a esta realidad cuando reclamaron que "muchas veces tengo que hacer las cosas de otra manera a como viene recogido en la guía, porque la realidad que me encuentro en clase no se adecúa a lo que figura en la guía docente. Incluso el sistema de evaluación que tengo establecido no es el que puedo utilizar" (UCAL).

Tal y como sintetizó uno de los profesores entrevistados, "la construcción del currículo es algo dinámico, no puedes prever en marzo, abril de un año lo que vas a hacer al año que viene. Yo, por ejemplo, que tengo clase en el segundo cuatrimestre, tengo que decir, ahora en marzo, abril o mayo o junio, lo que haré en enero, febrero del año que viene, sin conocer a qué alumnos, ni cuántos ni qué clase de alumnos" (ECSJ2).

\section{Contenido y estructura de la guía docente}

A la hora de valorar el modelo de guía docente que se viene empleando en la Universidad de La Laguna, el profesorado entrevistado consideró que era una herramienta útil para ofrecer al alumnado información básica sobre distintos aspectos de la asignatura. Efectivamente, desde la perspectiva de los docentes entrevistados, la guía docente recogió datos que eran de interés para el alumnado, tales como "la dirección del profesor, el contacto vía teléfono oficial, correo electrónico, lugar donde está ubicado, horarios de tutoría, horarios de clase" (EAH2).

No obstante, en lo que concierne a la parte más didáctica y académica de la guía docente, algunos de los entrevistados consideraron que "sobran apartados" (EC2). 
Concretamente, uno de los docentes señaló que la guía docente era una herramienta "muy tipificada; tienes que seguir paso por paso y de forma muy estricta todo lo que te indican" (EAH2"). Las opiniones del profesorado también reflejaron que la veían "demasiada amplia. Hay guías docentes, a lo mejor, de diez páginas, más o menos, entonces yo encuentro demasiada información totalmente superflua que no sirve para ti ni para el alumno a la hora de la práctica" (EAH2). En consecuencia, entendieron que "el exceso de información no favorece a que el alumno siga consultando la guía" (ECSJ1). Así, en el grupo de discusión se planteó que "la guía docente tendría que ser un documento más sencillo, tanto en sus funciones como en su estructura de cara al alumnado" (DEC).

De los apartados de la guía docente, uno de los más controvertidos fue el del cronograma. Con relación a ello, el profesorado entrevistado consideró que "el cronograma es lo que a veces es más complicado" (EC1). Otros sugirieron que este apartado "lo relleno porque me obligan a rellenarlo, pero realmente a mí esa parte me sobra" (EC2).

Como contrapartida al modelo de guía docente actual, algunos de los profesores entrevistados propusieron cambios de cara a mejorar esta herramienta sugiriendo que "me quedaría sobre todo con la metodología, que es importante, con los contenidos, evidentemente" (EAH1). Otro docente comentó que "lo fundamental es el temario, el contenido y la evaluación, todo lo demás me parece burocrático" (ECS1).

La simplificación de apartados se abordó también en el grupo de discusión, reconociendo la importancia de este recurso, pero demandando que las guías docentes fueran "documentos prácticos, útiles de verdad" (DEC). De manera más específica, se propuso "una guía en la cual aparecieran las competencias más importantes de la asignatura, las mínimas posibles, los tópicos a desarrollar como contenidos y algunos criterios de evaluación" (ECSJ2).

\section{Cumplimiento de la propuesta recogida en la guía docente}

Respecto al grado de cumplimiento de la propuesta recogida en la guía docente, el análisis de la información obtenida a partir de las entrevistas y el grupo de discusión, sugirió que, por un lado, había un grupo de docentes que cumplieron con lo establecido en el documento y, por otro, había profesores que no mantenían la correspondencia entre lo que se había establecido y determinado en la guía docente con la práctica educativa.

En relación con el profesorado que manifestó cumplir con la guía docente, comentaron que "en general sí se cumple... otra cuestión es la profundidad que se trate cada uno de ellos" (EIA1). Otro de los docentes señaló que "las mayores correspondencias se dan entre el contenido, lo doy íntegro, ahí no hay ningún tipo de desviación y en lo que se refiere al sistema evaluativo" (ECS1).

Por el contrario, aquellos docentes que no cumplieron con lo establecido en la guía argumentaron que había que tener en cuenta que "la guía docente la hacemos con un año de antelación, entonces hay cosas que son imponderables y complicado de ajustar" (EIA2). Esta falta de correspondencia también vino determinada por el propio diseño de la guía docente. De hecho, algunos de los informantes clave que fueron entrevistados indicaron que había guías docentes con "no sé cuántas competencias... y eso es imposible de abordarlo" (ECSJ1).

Otro profesor comentó la dificultad que tuvo cuando asumió la docencia en una asignatura y se encontró con un planteamiento formativo que no compartía. En esta 
línea, señaló que "imparto una asignatura donde los temas no los puedo tocar y es que no tienen nada que ver con lo que se tendría que dar en esa materia” (EC2).

En el grupo de discusión también se puso de manifiesto la disyuntiva existente entre la propuesta recogida en las guías docentes y la práctica educativa. Uno de los participantes comentó que "en uno de los procesos de acreditación, se detectó una guía en la que se recogía un contenido y unos criterios de evaluación que no tenían nada que ver con el título; como si se tratara de una guía docente sobre cine y el contenido que se recogía era sobre radio" (UCAL).

En cuanto a esta dimensión, el estudiante que participó en el grupo de discusión indicó que "la misma asignatura en Educación Infantil, por la mañana dan contenidos de sociales y en el grupo de tarde se habla de otros" (EU). Por estos motivos, se señaló que las comisiones de curso constituyen un espacio importante para plantear este tipo de problemas y disfunciones. Sin embargo, este mismo estudiante sugirió que otro problema era que a las comisiones de curso "no acuden todos los profesores; he ido a comisiones que se han tenido que anular porque somos tres personas” (EU).

\section{Discusión y conclusiones}

La finalidad principal de este estudio era analizar el modelo de guía docente que actualmente se viene empleando en la Universidad de La Laguna. Este proceso de revisión del modelo de guía docente no se puede aislar del contexto general en el que se desarrolla actualmente la enseñanza universitaria, donde se han producido cambios muy significativos en los últimos tiempos que, como señala Zabalza (2003), han venido a resaltar, aún más, algunas competencias del profesorado, como la planificación de la enseñanza. Siguiendo a Yaniz (2006), planificar y utilizar de manera efectiva distintas estrategias para diseñar y desarrollar con éxito la enseñanza, es una de las competencias docentes más importantes para afrontar el reto de la Convergencia Europea.

A pesar de este reconocimiento, el modelo formativo del EEES ha impuesto, como se desprende de los datos analizados, un diseño de la enseñanza bastante rígido y técnico, que restringe la capacidad del profesorado a la hora de planificar la enseñanza de las asignaturas. El modelo basado en el desarrollo de competencias (Yaniz y Villardón, 2006) determina una serie de decisiones que arrancan desde la memoria de verificación de la titulación, que limitan la autonomía y la creatividad del profesorado. De esta manera, lo que refleja el programa de la asignatura no siempre se corresponde con la propuesta del docente, dado que gran parte de la planificación viene predeterminada.

Desde la aprobación del Real Decreto 1393/2007, que reguló en España la enseñanza universitaria en el marco del EEES y todos los que le han sucedido, se requería como una parte de la planificación de los planes de estudio universitarios, la descripción de los módulos o materias de enseñanza-aprendizaje que conformaban el plan de estudios (art. 5.2 del anexo I). Esto implicaba el diseño de las guías docentes de las asignaturas (García, 2008) y en las distintas instituciones universitarias se viene cumpliendo con este requisito, de modo que se aprobó un modelo de guía docente cercano al espíritu de la Convergencia, común para todas las titulaciones.

Desde un enfoque institucional, el modelo cumple con los requisitos administrativos. Desde la perspectiva académica, se vienen planteando en los últimos tiempos algunas discrepancias sobre la utilidad de la guía docente para el alumnado y, 
sobre todo, se viene cuestionando si se trata de un recurso que verdaderamente guíe y oriente la práctica educativa. Precisamente en el modelo del EEES, el estudiante es el principal protagonista de la enseñanza y la guía docente tiene que tener en cuenta este requisito. Pensamos como Bozu y Cantó (2009), que la guía docente tiene que estar diseñada y pensada para que el alumnado sea el protagonista del aprendizaje. El estudiante es quien aprende y durante ese proceso, necesita orientación, referencias, pautas, etc. Esa es una función importante que debería desempeñar la guía docente: servir de referencia al alumnado durante el tiempo que dura una asignatura, de lo que debe aprender y de cómo se va a trabajar para conseguirlo. En este sentido compartimos con López y Crisol (2012) y García y De la Cruz (2014) la idea de que la guía docente ha de facilitar el trabajo independiente y el desarrollo de la actividad cognoscitiva en el alumnado.

A partir de los resultados obtenidos, se pone de manifiesto que la guía docente es un instrumento básico en el modelo actual de la enseñanza universitaria, especialmente valorado y utilizado en los procesos de verificación de las distintas titulaciones. Un criterio fundamental que emplea la ANECA en la acreditación y evaluación de las titulaciones es la adecuación de las guías docentes, tanto en lo que respecta a su diseño, como a su puesta en práctica. De ahí el esfuerzo que se viene realizando en el conjunto de las universidades españolas, para que las guías docentes cumplan con los requisitos y se consiga la acreditación y certificación de las titulaciones.

Sin embargo, a través del estudio se refleja también la escasa incidencia que las guías docentes tienen sobre el proceso formativo. Como se ha comprobado, el uso de la guía docente queda limitado, fundamentalmente, al primer día de clase, cuando se informa al alumnado de los elementos centrales de la asignatura. Pero no se percibe que sea un recurso que esté presente de manera permanente a lo largo del curso, alimentando y guiando el proceso de enseñanza-aprendizaje del alumnado. De ahí que se pueda concluir resaltando que la guía docente tiene más importancia como documento administrativo, que como recurso pedagógico y didáctico. Es decir, la guía docente no realiza realmente la función de "guía" del proceso formativo.

A ello puede contribuir la falta de identificación del profesorado con la guía docente, que en muchos casos le llega como un documento elaborado por otras personas, derivado técnicamente de la memoria del título que diseñó una o varias comisiones años atrás y con una orientación de la materia que para nada se ajusta a su modelo docente. Esta realidad puede ayudar a entender que, en muchos casos, la guía docente es simplemente un documento administrativo, que el profesorado entrega porque es de obligado cumplimiento, pero que no ofrece su propuesta adaptada a la realidad de la asignatura y a los procedimientos de aprendizaje que como docente considera más adecuados para los estudiantes que tiene a su cargo. En este sentido, es necesario que sean las áreas de conocimientos y los Departamentos quienes asuman la revisión más profunda y detenida de los programas y de los contenidos que se imparten en las asignaturas. Se debe tener presente que las asignaturas no son propiedad de un profesor, sino que corresponde al área determinar qué contenidos se deben trabajar en la misma, con independencia del docente que se encargue de desarrollarla. Esto sigue siendo un problema que no se ha logrado resolver, dado que, por un lado, se aprueba la guía docente y, por otro lado, el profesorado desarrolla su versión particular de la asignatura. A este proceso se le debería dedicar mayor atención cada año, llevando a cabo una verdadera revisión de los programas y abriendo espacios para un debate en profundidad sobre los contenidos que se deberían abordar según el área. Las propuestas 
que surjan de este análisis deberían trasladarse a la memoria de verificación, mediante los modifica correspondientes.

Resultó significativa la percepción sobre las guías docentes que tuvo el estudiante que participó en el estudio, considerándolas como una herramienta de escaso valor. Solo destacó su utilidad para encontrar información sobre aspectos muy específicos, como el nombre del profesado, los horarios, el sistema de evaluación, etc. y que se emplea en momentos concretos, como al elegir asignaturas o turnos.

Con relación al desajuste entre lo planificado en la guía docente y el proceso real de la enseñanza en el aula, resaltar las dificultades apuntadas por el profesorado a la hora de planificar algunos apartados de la guía docente. El cronograma, la metodología o sistema de evaluación es difícil concretarlos con tanta antelación, sin conocer el número de estudiantes, las características del grupo, etc. En este sentido, apartados de la guía como el cronograma se considera un elemento poco relevante y que se debería eliminar (es difícil prever con tanta antelación, en el mes de mayo/junio del curso anterior, que temas se van a impartir en cada periodo del curso siguiente). Por el contrario, otros apartados como la descripción de lo que aporta la asignatura al perfil del título no aparecen recogidos en el modelo de guía docente de la ULL y es recomendable su incorporación, dado que es un buen referente para la orientación del alumnado.

A modo de conclusiones y como derivación de este estudio, en relación a las guías docentes sería necesario:

- Aprobar procedimientos más ágiles para que las correcciones que se solicitan a la ANECA mediante modificaciones de las memorias de verificación del título, se aprueben e incorporen de manera más rápida.

- Diseñar un modelo de guía docente más sencillo, en el que se recojan los apartados básicos (resultados de aprendizaje, competencias, contenidos, sistema de evaluación y bibliografía) y se eliminen otros poco significativos como la temporalización o cronograma.

- Lograr que las áreas de conocimiento se impliquen y tengan mayor protagonismo en la determinación de los contenidos básicos de cada asignatura.

- Permitir una mayor autonomía al profesorado a la hora de hacer suya la guía docente y desarrollar su propuesta metodológica, después de garantizar que se cumple con los requisitos básicos del programa de cada asignatura.

- Sensibilizar al profesorado acerca de la importancia y conveniencia de que las guías docentes estén presentes a lo largo del curso, como un elemento integrado en el proceso formativo.

- Fomentar en el alumnado la utilización de la guía docente, como un referente y recurso orientativo del proceso de enseñanza-aprendizaje.

\section{Referencias}

Arias-Gundín, O., Fidalgo, R., y García, J.N. (2008). El desarrollo de las competencias transversales en Magisterio mediante el ABP y el Método del Caso. Revista de Investigación Educativa, $26 \quad$ (2), 431-444. Retrieved from https://revistas.um.es/rie/article/view/94011 
Bozu, Z., y Canto, P. (2009). El profesorado universitario en la sociedad del conocimiento: competencias profesionales docentes. Revista de Formación e Innovación Educativa Universitaria, 2 (2), 87-97. Retrieved from http://refiedu.webs.uvigo.es/Refiedu/Vol2_2/REFIEDU_2_2_4.pdf

Cano, M., Tomás, R., García, J., Bañón, L., Riquelme, A.J., y Murcia, I. (2016). Evaluación de la implementación de las guías docentes de las asignaturas del ámbito de la Ingeniería del Terreno del Máster en Ingeniería Geológica. En J.D. Álvarez, S. Grau, y M.T. Tortosa (coord.), Innovaciones metodológicas en docencia universitaria: resultados de investigación (417-434). Alicante: Servicio de publicaciones de la Universidad de Alicante.

Declaración de Bolonia. Declaración conjunta de los Ministros Europeos de Educación reunidos en Bolonia, junio 1999. Consultado el 27 de junio de 2018 en http://www.eees.es/pdf/Declaracion_Bolonia.pdf

Díez, C., Pacheco, D., y García, J. (2008). Las habilidades sociales en el marco del EEES. En M. Hijano del Río. Las titulaciones de educación ante el Espacio Europeo de Educación Superior: análisis de experiencias (239-247). Málaga: Aljibe.

Ercikan, K., y Roth, W. (2006). What good is polarizing research into qualitative and quantitative? Educational Researcher, 35 (5), 14-23. doi: 10.3102/0013189X035005014

Fernández, A., y Fernández, M. (2007). Enfoques de evaluación educativa en la enseñanza universitaria. En M. López. Evaluación de los procesos de enseñanza aprendizaje en la universidad y su adaptación al Espacio Europeo de Educación Superior (29-46). Granada: Editorial Universidad de Granada.

Fidalgo, R., y García, J. (2007). Las directrices del Espacio Europeo de Educación Superior en el marco legislativo del sistema universitario español. Aula Abierta, 35, 35-48. Retrieved from https://www.researchgate.net/profile/Raquel_Fidalgo/publication/28239455_Las _directrices_del_Espacio_Europeo_de_Educacion_Superior_en_el_Marco_Legi slativo_del_Sistema_Universitario_Espanol/links/0deec529ed992ccd47000000/ Las-directrices-del-Espacio-Europeo-de-Educacion-Superior-en-el-MarcoLegislativo-del-Sistema-Universitario-Espanol.pdf

García, E.C., y Deltell, L. (2012). La guía docente: un reto en el nuevo modelo de educación universitaria. Estudios sobre el mensaje periodístico, 18, 357-364.

García, I., y De la Cruz, G. (2014). Las guías didácticas: recursos necesarios para el aprendizaje autónomo. Revista Edumecentro, 6 (3), 162-175. Retrieved from http://scielo.sld.cu/scielo.php?script=sci_arttext\&pid=S207728742014000300012

García, L. (2014). La Guía Didáctica. Contextos Universitarios Mediados, 14, 5.

García, M. (2008). Guías docentes de asignaturas de Grado en el EEES. Editum: Universidad de Murcia.

González, C., Gilar, R., Mañas, C., y Bermejo, M. (2005). Estudio de los elementos que configuran la guía docente, como material de trabajo, en la adaptación al sistema europeo de créditos (ECTS) para la docencia universitaria. III Jornades de 
Xarxes d'Investigació en Docència Universitària: la configuració de l'Espai Europeu d'Educació Superior. Alicante: Universidad de Alicante.

Grinell, R. (1997). Social work research \& evaluation: Quantitative and qualitative approaches. Illinois: Peacock Publishers.

Hernández, R., Fernández-Collado, C., y Baptista, P. (2006). Metodología de la investigación. México: McGrawHill.

Huber, G. (1992). Qualitative Analyse: Coputereinsatz in der Sozialoforschung. Munich: R. Oldenbourg Verlag.

López, M.A., y Crisol, E. (2012). Las guías de aprendizaje autónomo como herramienta didáctica de apoyo a la docencia. Escuela Abierta, 15, 9-31. Retrieved from https://dialnet.unirioja.es/servlet/articulo?codigo $=4078711$

Marcelo, C., Yot, C., Mayor, C., Sánchez, M., Murillo, P., Rodríguez, J. M., y Pardo, A. (2014). Las actividades de aprendizaje en la enseñanza universitaria: ¿hacia un aprendizaje autónomo de los alumnos? Revista de Educación, 363, 34-359. doi: 10-4438/1988-592X-RE-2012-363-191

Mas, O. (2012). Las competencias del docente universitario: la percepción del alumno, de los expertos y del propio protagonista. Revista de Docencia Universitaria, 10

$\begin{array}{lr}\text { (2), } & \text { Retrieved } \\ \text { https://dialnet.unirioja.es/servlet/articulo?codigo=4021101 }\end{array}$

McMillan, E., y Schumacher, S. (2005). Investigación Educativa. Madrid: Pearson (5 Edición).

Miles, M. B., y Huberman, A. M. (1994). Qualitative data analysis: an expanded sourcebook. Thousand Oaks: Sage.

Morón, J.A., y Pedrero, E. (2012). Orientando el proceso de enseñanza-aprendizaje en la asignatura de educación para la salud: la guía docente en el Espacio Europeo de Educación Superior. Revista UPO Innova, I, 375-390. Retrieved from https://www.researchgate.net/publication/279653198_Orientando_el_proceso_d e ensenanzaaprendizaje_en_la_asignatura_de_educacion_para_la_salud_la_guia_docente_en _el_espacio_europeo_de_educacion_superior

Real Decreto 1393/2007, de 29 de octubre, por el que se establece la ordenación de las enseñanzas universitarias oficiales.

Rogers, G., y Bouey, E. (2005). Participant observation. En R.M. Grinnell, y Y.A. Unrau (eds), Social Work: Research and evaluation. Quantitative and qualitative approaches. Nueva York: Oxford University Press.

Salinas, B., y Cotillas, C. (2005). Elaboración de la Guía Docente para la Convergencia Europea. Valencia: Servei de Formació Permanent de la Universitat de València.

Sánchez, M., Ruiz, C., y Pascual (2001). La guía docente como eje del proceso de enseñanza aprendizaje. Bordón, 63 (2), 53-64. Retrieved from https://recyt.fecyt.es/index.php/BORDON/article/view/28971

Sanz, S., Ruiz, C., y Pérez, I. (2014). El profesor universitario y su función docente. ESPACIO $i+D$, Innovación más Desarrollo, III (5), 97-112. Retrieved from 
http://www.espacioimasd.unach.mx/articulos/num5/El_Profesor_universitario_y _su_funcion_docente.php

Strauss, A., y Corbin, J. (2002). Bases de la investigación cualitativa. Técnicas y procedimientos para desarrollar la teoría fundamentada. Colombia: Universidad de Antioquía.

Suárez, M. (2005). El grupo de discusión. Una herramienta para la investigación cualitativa. Barcelona: Laertes

Yaniz, C. (2006). Planificar la enseñanza universitaria para el desarrollo de competencias. Educatio Siglo XXI, 24, 17 - 34. Retrieved from https://revistas.um.es/educatio/article/view/151

Yániz, C., y Villardón, L. (2006). Planificar desde competencias para promover el aprendizaje. Bilbao: Universidad de Deusto.

Zabalza, M. A. (2003). Competencias docentes del profesorado universitario. Calidad y desarrollo profesional. Madrid: Narcea.

Fecha de recepción: 04/05/2018

Fecha de revisión: 28/10/2018

Fecha de aceptación: 17/01/2019 